\title{
Measurements of the top-quark properties at CMS
}

\author{
Efe YAZGAN** \\ Department of Physics and Astronomy, University of Ghent, Proeftuinstraat 86, B-9000 Ghent, \\ Belgium \\ E-mail: efe.yazgan@cern.ch
}

\begin{abstract}
Measurements of several top-quark properties, obtained from the CMS data collected in 2011 and 2012 at centre-of-mass energies of 7 and $8 \mathrm{TeV}$ are presented. The results include measurements of the top pair charge asymmetry, the W helicity in top quark decays and the search for anomalous couplings, the top quark charge, and of the $t \bar{t}$ spin correlation. The fraction of top quarks decaying into a W-boson and a b-quark relative to all top quark decays, $\mathscr{R}=\mathscr{B}(t \rightarrow W b) / \mathscr{B}(t \rightarrow W q)$, as well as, the cross sections of ttbar events produced in association with a photon or a $\mathrm{W}$ or a $\mathrm{Z}$ boson are also presented.
\end{abstract}

XXI International Workshop on Deep-Inelastic Scattering and Related Subject -DIS2013, 22-26 April 2013

Marseilles, France

\footnotetext{
* Speaker.

${ }^{\dagger}$ On behalf of the CMS Collaboration
} 


\section{Introduction}

The most massive particle, the top quark, has a shorter lifetime than the hadronization timescale. This makes its bare quark properties observable. The meaurements presented in this note use the data collected with the CMS [1] detector during 2011 and 2012 data taking periods with protonproton collisions at $\sqrt{s}=7$ and $8 \mathrm{TeV}$, respectively.

\section{2. $t \bar{t}$ Charge Asymmetry}

Tevatron experiments observed a deviation of $\sim 2-3 \sigma$ from the standard model (SM) $t \bar{t}$ forward-backward asymmetry $[2,3]$. The asymmetry occurs in $t \bar{t}$ production through quarkantiquark annihilation. The antiquark at the LHC is a sea quark, therefore, on average the quark has a larger momentum fraction. This results in an excess of top quarks in forward directions. Therefore, the charge asymmetry $\left(A_{C}\right)$ defined using the rapidity difference of the top and the antitop quark $\left(\Delta|y|=\left|y_{t}\right|-\left|y_{\bar{t}}\right|\right)$ is a reasonable choice for the LHC. In the lepton+jets channel, the measurement resulted in $A_{C}=0.004 \pm 0.010$ (stat) \pm 0.011 (sys.) [4] compatible with the SM prediction at NLO, $0.0115 \pm 0.0006$ [5]. In the dilepton channel, the inclusive $A_{C}$ is measured to be $0.050 \pm 0.043$ (stat) $)_{-0.039}^{+0.010}$ (sys.). Inclusive $A_{C}$ is also measured using lepton pseudo-rapidities and is found to be $0.010 \pm 0.015$ (stat) \pm 0.006 (sys.) [6]. These are in agreement with the NLO predictions of $0.0123 \pm 0.0005$ and $A_{C}(\Delta|\eta|)=0.0156 \pm 0.0007$ [7]. Figure 1 displays $(\Delta|y|)$, rapidity $\left(\left|y_{t \bar{t}}\right|\right)$, transverse momenta $\left(p_{T, t \bar{t}}\right)$, and the invariant mass $\left(m_{t \bar{t}}\right)$ of the $t \bar{t}$ system. $\left|y_{t \bar{t}}\right|$ depends on the ratio of events with $q \bar{q}$ and $g g$ initial states and at small rapidities, gluon fusion dominates. Therefore, $A_{C}$ increases with increasing $\left|y_{t \bar{t}}\right|$ [5] (see Figure 1 upper right). Interferences between the Born and the box diagrams and also between the initial- and final-state-radiation cause the (anti)top quark direction to be correlated to the initial state (anti)quark. In the former case, there is a positive contribution to $A_{C}$ and a negative contribution in the latter. In the presence of initialor final-state-radiation the $t \bar{t}$ system has a higher $p_{T}$, hence there is more negative contribution to $A_{C}$ for such events [5] (see Figure 1 lower left). At high invariant mass of the $t \bar{t}$ system, $m_{t \bar{t}}$, number of $t \bar{t}$ events with $q \bar{q}$ production increases and therefore $A_{C}$ increases (see Figure 1 lower right). However, if new heavy particles have a role in $t \bar{t}$ production, they can interfere with the SM processes and this might result in a different dependence on $m_{t \bar{t}}$. The distributions in Figure 1 are also compared to the distributions predicted by a model with an effective axial-vector coupling of the gluon $[8,9]$ that could describe the depence of forward-backward asymmetry vs $m_{t \bar{t}}$ observed at the Tevatron. None of the distributions in Figure 1 shows a deviation from the SM expectations however the uncertainties are still large.

\section{W Boson Helicities in Top Quark Decays and Anomalous Couplings}

Partial witdh of the top quark can be parametrized in terms of left-handed $\left(\mathrm{F}_{L}\right)$, right-handed $\left(F_{R}\right)$, longitudinal $\left(F_{0}\right) \mathrm{W}$ boson helity fractions and the $\theta^{*}$ angle between the momentum of the d-type fermion in the $W$ rest frame and of the $W$ in the top quark rest frame. $W$ helicities in $t \bar{t}$ events in dilepton and lepton+jets final states are measured [10,11]. Measurements at $\sqrt{s}=7 \mathrm{TeV}$ made by CMS and ATLAS are also combined [12]. Individual and combined measurements are 

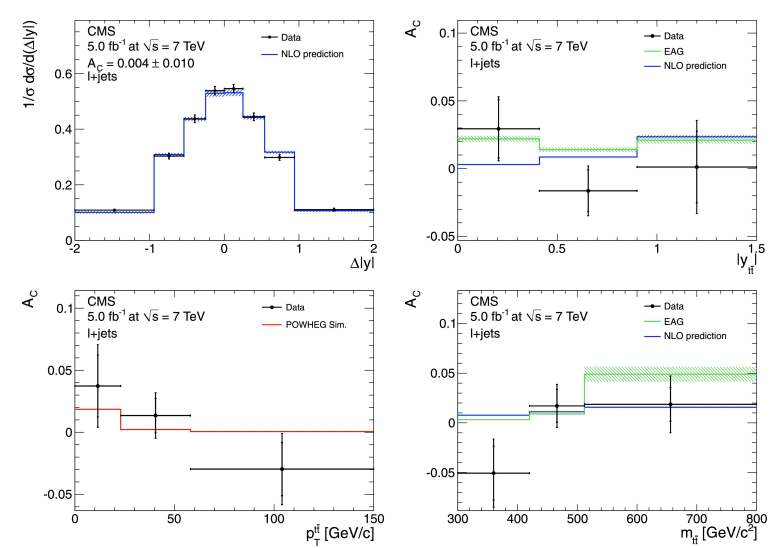

Figure 1: Unfolded inclusive $\Delta|y|$ distribution (upper left), $A_{C}$ vs $\left|y_{t \bar{t}}\right|$ (upper right), $p_{t \bar{t}}$ (lower left), and $m_{t \bar{t}}$ (lower right). The measurements are compared to SM NLO calculations and to the predictions of an effective model.

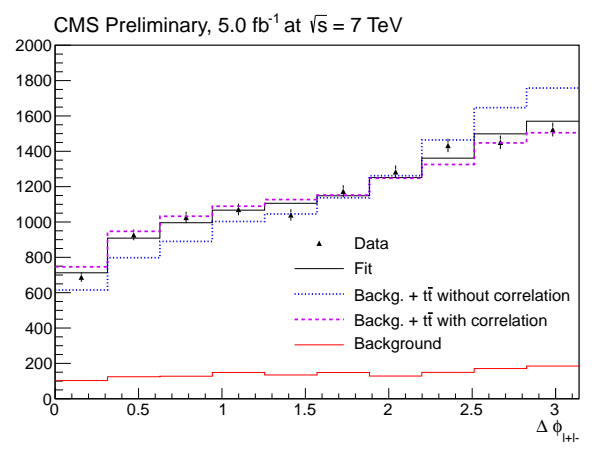

Figure 2: Result of the fit (solid line) performed on data (triangles) after the combination of the three channels. The data are also compared to the $\Delta \Phi_{\ell^{+} \ell^{-}}$ distribution of $t \bar{t}$ pair events with and without spin correlation. The background components are also shown.

found to be consistent with each other and with SM predictions at NNLO QCD. Measurement of W boson helicity fractions is also performed in single-top topologies [13] in the $\mu+$ jets final state at $\sqrt{s}=7$ and $8 \mathrm{TeV}$. The helicity fractions are obtained from likelihoods with reweighted signals including all processes involving the top quark. The measurements performed at $\sqrt{s}=7$ and $8 \mathrm{TeV}$ are combined yielding $F_{0}=0.713 \pm 0.114$ (stat) \pm 0.023 (syst), $F_{L}=0.293 \pm 0.069$ (stat) \pm 0.030 (syst) and $F_{R}=-0.006 \pm 0.057$ (stat) \pm 0.027 (syst). The measurements are consistent with the SM predictions and the measurements in the $t \bar{t}$ channels. These measurements are also used to set limits on the real part of the anomalous $W t b$ couplings.

\section{Top Quark Charge}

Being the electroweak isospin partner of the b-quark, top quark has an electric charge of $+2 / 3 e$. A measurement is made testing two different top quark charge hypotheses in the muon+jets final state at $\sqrt{s}=7 \mathrm{TeV}$ [14]. For the measurement, charge correlations between muons from $\mathrm{W}$ boson decays and soft muons from B-hadron decays in b-jets are utilized to constrain the top quark charge. Using a normalized asymmetry variable, the top quark charge hypothesis of $-4 / 3 e$ against $+2 / 3 e$ is tested. The measurement yielded an asymmetry of $A=0.97 \pm 0.12$ (stat.) \pm 0.31 (sys.) in agreement with the SM expectation of $A=+1$.

\section{5. $t-\bar{t}$ Spin Correlation}

The spin-decorrelation timescale, $m_{t} / \Lambda_{Q C D}^{2} \sim 3 \times 10^{-21} \mathrm{~s}$, is larger than the hadronization time scale. Therefore spin effects propagate to the decay products. In the dilepton final state, the azimuthal angle between the opposite charge leptons, $\left|\Delta \phi_{\ell^{+} \ell^{-}}\right|$, in the laboratory frame is sensitive to the $t-\bar{t}$ spin correlation. This variable can be measured precisely without top quark reconstruction. At $\sqrt{s}=7 \mathrm{TeV}$, the $t-\bar{t}$ spin correlation in the dilepton channel is extracted from a template fit 
to the $\left|\Delta \phi_{\ell^{+} \ell^{-}}\right|$distribution [15]. Three different templates are used; simulated $t \bar{t}$ events assuming the SM, simulated $t \bar{t}$ events without spin correlation and a template for the background events. A binned likelihood fit to data is used to simultaneously fit the $e^{+} e^{-}, \mu^{+} \mu^{-}$and $e^{ \pm} \mu^{\mp}$ channels to determine the fraction of events with SM spin correlation, $f=N_{S M} /\left(N_{S M}+N_{\text {uncor }}\right)$. The measurement yielded $f=0.74 \pm 0.08$ (stat.) \pm 0.24 (sys.) consistent with the SM expectation of $f^{S M}=1$. The result of this fit performed on data after the combination of the three channels is shown in Figure 2. The data are also compared to the $\left|\Delta \phi_{\ell^{+} \ell^{-}}\right|$distributions obtain from $t \bar{t}$ events with and without spin-correlation. The fraction of events with SM spin correlation, $f$, can be converted to a measurement of the spin correlation coefficient $A$. In the helicity basis, the measurement yields $A_{\text {hel }}^{\text {meas }}=0.24 \pm 0.02$ (stat.) \pm 0.08 (sys.) consistent with the SM prediction at NLO of $A_{\text {hel }}^{S M}=0.31$ [16]. The spin correlation is also studied using other variables and at different $m_{t \bar{t}}$ values [15]. All measurements are found to be consistent with the SM predictions.

\section{Measurement of the Ratio $\mathscr{R}=\mathscr{B}(t \rightarrow W b) / \mathscr{B}(t \rightarrow W q)$}

The top quark decays to a $\mathrm{W}$ boson and a b quark practically with a branching ratio of $\sim 100 \%$. Decays to other down-type quarks are suppressed in the CKM matrix. A measurement of $\left|V_{t b}\right|$ is made by measuring the branching fraction ratio $\mathscr{R}=\mathscr{B}(t \rightarrow W b) / \mathscr{B}(t \rightarrow W q)$ in the $t \bar{t}$ di-lepton final state using $\sqrt{s}=8 \mathrm{TeV}$ data [17]. $\mathscr{R}$ is extracted from a profile likelihood fit to data-driven analytic probability models of signal purity, number of reconstructed tops in different jet categories and number of b-tags. The fit gave $\mathscr{R}=1.023_{-0.034}^{+0.036}$ (stat+syst). Imposing $\mathscr{R} \leq 1$, a lower limit for $\mathscr{R}$ is derived to be $\mathscr{R}>0.945$ at $95 \%$ C.L. With the assumption of CKM unitarity and existence of three generations, the measurement is converted to a measurement of $\left|V_{t b}\right|$ yielding $1.011_{-0.017}^{+0.018}$ and a lower limit is derived to be $\left|V_{t b}\right|>0.972$ at $95 \%$ C.L. The results obtained for $\mathscr{R}$ and $\left|V_{t b}\right|$ are consistent with the SM predictions and represent the most precise measurement of $\mathscr{R}$ and the most stringent direct limit on $\left|V_{t b}\right|$.

\section{Vector Boson Production Associated with Top-Antitop Pairs}

Measurement of vector boson production in association with $t \bar{t}$ pairs provides a test of the SM top quark-vector boson couplings. Measurement of these couplings are important for new physics searches and also the measurements of the Higgs boson in the $t \bar{t} H$ process. The cross section measurements of the $t \bar{t} V$ processes are made using the same-sign dilepton for $t \bar{t} W$ and $t \bar{t} Z$ and trilepton signature for $t \bar{t} Z$ process at $\sqrt{s}=7 \mathrm{TeV}$ [18]. The cross section for $t \bar{t} V$ is measured to be $0.43_{-0.15}^{+0.17}(\text { stat })_{-0.07}^{+0.09}($ syst $) \mathrm{pb}$ and $t \bar{t} Z$ to be $0.28_{-0.11}^{+0.14}(\text { stat })_{-0.03}^{+0.06}($ syst $)$ pb consistent with the SM NLO calculations $[19,20]$. The $t \bar{t} Z$ measurements represent the first direct measurements of the top quark-Z boson coupling.

\section{Conclusions}

Measurements of top quark properties are providing thorough tests of the SM. All top quark properties measurements at the LHC are in good agreement with the SM predictions. 


\section{References}

[1] CMS Collaboration, The CMS experiment at the CERN LHC, JINST 3 (2008) S08004.

[2] CDF Collaboration, Measurement of the top quark forward-backward production asymmetry and its dependence on event kinematic properties, PRD 87 (2013) 092002 [arXiv:1211.1003 [hep-ex]].

[3] D0 Collaboration, Forward-backward asymmetry in top quark-antiquark production, PRD 84 (2011) 112005 [arXiv:1107.4995 [hep-ex]].

[4] CMS Collaboration, Inclusive and differential measurements of the t $\bar{t}$ charge asymmetry in proton-proton collisions at $\sqrt{s}=7 \mathrm{TeV}$, PLB 717 (2012) 129 [arXiv:1207.0065 [hep-ex]].

[5] J. H. Kuhn, and G. Rodrigo, Charge Asymmetries of top quarks at hadron colliders revisited, JHEP 01 (2012) 063 [arXiv:1109.6830 [hep-ph]].

[6] CMS Collaboration, Top charge asymmetry measurement in dileptons at $7 \mathrm{TeV}$, CMS-PAS-TOP-12-010 (2012).

[7] W. Bernreuther and Z-G. Si, Top quark and leptonic charge asymmetries for the Tevatron and LHC, PRD 86 (2012) 034026 [arXiv:1205.6580 [hep-ph]].

[8] E. Gabrielli, A. Racioppi, M. Raidal, Implications of the effective axial-vector coupling of the gluon on top-quark charge asymmetry at the LHC, PRD 85 (2012) 074021 [arXiv:1112.5885v1 [hep-ph]].

[9] G. Brooijmans, et al., Les Houches 2011: Physics at TeV colliders new physics working group report, (2012) [arXiv:1203.1488v2 [hep-ph]].

[10] CMS Collaboration, Measurement of the $W$ boson polarization in semileptonic top pair decays with the CMS detector at the LHC, CMS-PAS-TOP-11-020 (2011).

[11] CMS Collaboration, Measurement of the Whelicity in top pair production with dileptons at $7 \mathrm{TeV}$, CMS-PAS-TOP-12-015 (2012).

[12] ATLAS and CMS Collaborations, Combination of the ATLAS and CMS measurements of the W-boson polarization in top-quark decays, ATLAS-CONF-2013-033, CMS-PAS-TOP-12-025 (2013).

[13] CMS Collaboration, W-helicity measurement in single top events topology, CMS-PAS-TOP-12-020 (2012).

[14] CMS Collaboration, Constraints on the Top-Quark Charge from Top-Pair Events, CMS-PAS-TOP-11-031 (2011).

[15] CMS Collaboration, Measurement of Spin Correlations in t $\bar{t}$ events in the dilepton channels in $p p$ collisions at $\sqrt{s}=7 \mathrm{TeV}$, CMS-PAS-TOP-12-004 (2012).

[16] W. Bernreuther and Z.-G. Si, Distributions and correlations for top quark pair production and decay at the Tevatron and LHC, Nuc. Phys. B 837 (2010) 90 [arXiv:1003.3926 [hep-ph]].

[17] CMS Collaboration, Measurement of the ratio $B(t \rightarrow W b) / B(t \rightarrow W q), C M S-P A S-T O P-12-035$ (2012).

[18] CMS Collaboration, Measurement of associated production of vector bosons and top quark-antiquark pairs in pp collisions at $\sqrt{s}=7 \mathrm{TeV}$, PRL 110 (2013) 172002 [arXiv:1303.3239 [hep-ex]].

[19] J. M. Campbell and R. K. Ellis, $t \bar{t} W^{ \pm}$production and decay at NLO, JHEP 07 (2012) 052.

[20] M.V. Garzelli, A. Kardos, C.G. Papadopoulos, Z. Trocsanyi $t \bar{t} W^{ \pm}$and $t \bar{t} Z$ Hadroproduction at NLO accuracy in QCD with Parton Shower and Hadronization effect, JHEP 11 (2012) 056. 